\title{
Oocyte stem cells: fact or fantasy?
}

\author{
Corrina J Horan and Suzannah A Williams \\ Nuffield Department of Obstetrics and Gynaecology, University of Oxford, Women's Centre, John Radcliffe \\ Hospital, Oxford, United Kingdom \\ Correspondence should be addressed to S A Williams; Email: suzannah.williams@obs-gyn.ox.ac.uk
}

\begin{abstract}
For many decades, the dogma prevailed that female mammals had a finite pool of oocytes at birth and this was gradually exhausted during a lifetime of reproductive function. However, in 2004, a new era began in the field of female oogenesis. A study was published that appeared to detect oocyte-stem cells capable of generating new eggs within mouse ovaries. This study was highly controversial and the years since this initial finding have produced extensive research and even more extensive debate into their possibility. Unequivocal evidence testifying to the existence of oocyte-stem cells (OSCs) has yet to be produced, meanwhile the spectrum of views from both sides of the debate are wide-ranging and surprisingly passionate. Although recent studies have presented some convincing results that germ cells exist and are capable of creating new oocytes, many questions remain. Are these cells present in humans? Do they exist in physiological conditions in a dormant state? This comprehensive review first examines where and how the dogma of a finite pool was established, how this has been challenged over the years and addresses the most pertinent questions as to the current status of their existence, their role in female fertility, and perhaps most importantly, if they do exist, how can we harness these cells to improve a woman's oocyte reserve and treat conditions such as premature ovarian insufficiency (POI: also known as premature ovarian failure, POF).

Reproduction (2017) 154 R23-R35
\end{abstract}

\section{Introduction}

Male germ-line cells are produced continuously throughout adult life (Brinster 2007) but female oogenesis is less well understood. At birth, 500,0001,000,000 primordial follicles are estimated to be present (each containing a primary oocyte). However, this number declines rapidly with $\sim 25,000$ remaining in the ovaries by 38 years of age, and then decreasing ever more rapidly until $\sim 1000$ remain by $\sim 51$ years of age resulting in menopause (Hansen et al. 2008). With a woman ovulating once a month from puberty to menopause, less than 500 of these oocytes will ever be ovulated. However, recent controversy exists regarding the origin of these oocytes: are they all present at birth as has been thought for years, or does postnatal oogenesis occur in females as more recently proposed? Data is accumulating to indicate a pool of stem cells exists within mammalian ovaries and can be manipulated to produce oocytes in vitro. However, the function of any such stem cells in vivo is not understood and their natural function, if any, remains elusive.

\section{Origin of the debate}

These primordial germ cells appear under many guises in current research; often known as germ-line stem cells (Bukovsky et al. 2004, Gosden 2004, Byskov et al. 2005, Bukovsky 2011), ovarian stem cells (VirantKlun et al. 2008, Dunlop et al. 2013, Esmaeilian et al. 2015), putative stem cells (Bui et al. 2014), primordial germ cells (David 1974) and occasionally known as premeiotic germ cells (Niikura et al. 2009), ovarian germ stem cells (Patel et al. 2013) or oogonial stem cells (Anderson 2013, Imudia et al. 2013). In this article, they will be referred to as oocyte-stem cells (OSCs).

Initial studies of OSCs were few and far between, based on different species and generated conflicting ideas about their nature. Waldeyer, in 1870, stated that oocyte production ceases shortly after birth in female mammals, based on various studies of the ovaries of multiple mammals, including humans, dogs and cats (Table 1). Although widely quoted, the full transcript of this paper is in german, creating the potential for details to get lost in translation (Waldeyer 1870). This view was supported by Pearl and Schoppe in 1921 (Table 1) who declared that primary oocyte supply was not increased during adult life; their conclusions were based on counting visible oocytes from the ovaries of reproduction age fowl and also results on fowl from 8 years previously (Pearl \& Schoppe 1921). Although interesting, the physiology of fowl differs substantially from that of mammals. A quote from Pearl and Schoppe's 1921 paper states 'It has been held as a basic biological doctrine that during 
Table 1 Summary of initial findings regarding the finite nature of the germ cell pool.

\begin{tabular}{|c|c|c|}
\hline Authors & Year & Main findings \\
\hline Waldeyer & 1870 & $\begin{array}{l}\text { A collation of studies on many species indicating that oocyte production ceases shortly } \\
\text { after birth. }\end{array}$ \\
\hline Pearl and Schoppe & 1921 & $\begin{array}{l}\text { Counted visible oocytes from the ovaries of fowl of reproductive age. Concluded that } \\
\text { primary oocyte supply was not increased during adult life. }\end{array}$ \\
\hline Zuckerman & 1951 & $\begin{array}{l}\text { Review of previous evidence including rats and monkeys, concluding that neo-oogenesis } \\
\text { in mammals did not occur beyond a few days postnatally. }\end{array}$ \\
\hline Peters, Levy and Crone & 1962 & $\begin{array}{l}\text { Studied DNA synthesis within mouse oocytes. Concluded that it became insignificant at } \\
\text { embryonic day } 18 \text {. }\end{array}$ \\
\hline
\end{tabular}

the life of the individual there neither is nor can be any increase in the number of primary oocytes beyond those originally laid down when the ovary was formed' (Pearl \& Schoppe 1921). However, their research contradicts their conclusions as further oocytes could apparently be produced after mechanical damage to the fowl ovary; however, the potential source of these 'new' oocytes is not discussed in the paper. In 1923, Allen reviewed evidence supporting and opposing oocyte regeneration in adult rats, and investigated the possibility of oocyte production in adult mice (Allen 1923). The investigations were based on data from Arai in 1920 (Arai 1920) who studied rats with one ovary removed before puberty (semi-spayed); this provided evidence for equal ovarian function between healthy and semi-spayed rats, based on the total number of eggs obtaining maturity. They proposed that if no compensation mechanism were present, the semi-spayed rats would produce half of the number of mature eggs when compared with nonspayed rats. In order to research ovarian function further, Allen studied histologically defined mitotic cells within the ovarian surface epithelium of mouse ovaries that were either semi-spayed before puberty and after puberty, or non-spayed. The number of mitotic germ cells identified in semi-spayed mice correlated with different stages of the oestrous cycle, indicating a cyclic pattern of cell proliferation (Allen 1923). Allen concluded that the mitotic cells were markers of postnatal oogenesis. However, these mitotic cells could represent a compensatory mechanism of increased immature follicle development, namely the initial development and division of the theca and granulosa cells within the follicle, after removal of one ovary. Furthermore, this compensation may occur without any oogenesis. The cyclical pattern of mitotic activity within the ovary, whatever it represents, was supported by Bullough's lab in 1942 studying mitotic activity in the ovarian surface epithelium of the adult mouse throughout the oestrous cycle (Bullough 1942).

Despite these diverse findings from various groups over the previous 50 years, Zuckerman and coworkers published a paper in 1951 with conclusions that would become dogma for over 50 years (Zuckerman 1951) (Table 1). A review of the previous evidence was performed by Zuckerman, which included experiments involving irradiation of the ovary and the resulting ischaemic degeneration of the ovarian surface epithelium in rats and monkeys. The conclusion of the review was that neo-oogenesis in mammals did not occur postnatally beyond a few days and did not occur after irradiation. This paper marked a conclusive rejection of the idea of postnatal oogenesis that would predominate for over 50 years. The conclusions of Zuckerman were supported by Peters in 1962 (Table 1), who analysed DNA synthesis within mouse oocytes (Peters et al. 1962). Peters and coworkers investigated DNA synthesis in oocytes using a radioactive DNA precursor, in the form of tritium-labelled thymidine, injected at different days of gestation. The results revealed that germ cell DNA synthesis became insignificant at embryonic day 18 (E18) (Peters et al. 1962). However, this study did not study mice postnatally, which limits conclusions that can be drawn in relation to neo-oogenesis post birth.

This conclusion was widely accepted, however a different picture was found in ovaries of prosimian primates by Ioannou in 1967 (Ioannou 1967). Ovaries of adult prosimian primates Perodicticus, Galago and Loris animals were studied and oogonia undergoing mitosis or meiosis were analysed using two methods to identify oogonia: histological identification of cells in different stages of division; and the presence of DNAsynthesising germ cells using an autoradiographic technique. Both techniques demonstrated the presence of oogonia undergoing mitosis (mostly prophase and interphase), as well as oocytes at successive stages of meiosis up to diplotene (Ioannou 1967). Baker's group, also using an autoradiographic method to identify germinal cells within the ovaries of Loris primates, supported the conclusion that germinal cells are present and dividing, and therefore that post-natal oogenesis occurs (David 1974). However, identification of mitotic cells was limited to histological analysis of haematoxylin stained sections, with no specific staining for oocyte markers. The dividing cells could therefore be granulosa/theca cells, or other support cells present within the ovary.

\section{A new era}

Despite these findings in Loris primates, Zuckerman's conclusions prevailed and were essentially unchallenged until 2004, when Tilly's group published 
a paper refuting them entirely (Johnson et al. 2004). Johnson and coworkers studied follicle dynamics within mouse ovaries from embryonic development through to adulthood. Immature follicles were the focus, and were defined as primordial, primary or preantral according to the layers of surrounding cells and morphology. Follicle depletion was analysed by determining the numbers of immature follicles that were healthy, degenerating or contained a fragmented oocyte (classified as atretic), and resulted in data inconsistent with oogenesis terminating shortly after birth (Johnson et al. 2004). Tilly's group also studied oogenesis in post-natal mouse ovaries through a variety of methods; BrdU and mouse Vasa homolog ( $\mathrm{MVH}$; a germ cell marker) detection was used to identify presumptive oocyte stem cells. Expression of mitotic gene markers indicated that these cells were dividing. In addition, growth of GFP-labelled OSCs into follicles when implanted into wild-type mouse ovaries provided evidence for their potential function in vivo. These results supported the following conclusion 'adult mouse ovaries contain stem cells capable of generating new eggs that can be fertilised to produce viable offspring.' (Johnson et al. 2004).

The claim of post-natal mammalian oogenesis polarised the scientific community, with many studies supporting the presence of OSCs (Bukovsky et al. 2005, Johnson et al. 2005a, Virant-Klun et al. 2008, Niikura et al. 2009, Zou et al. 2009, Pacchiarotti et al. 2010, Parte et al. 2011, Zhang et al. 2011, White et al. 2012, Zhou et al. 2014), whereas many others believed the data had been misinterpreted refuting the finding of OSCs (Bristol-Gould et al. 2006, Liu et al. 2007, Byskov et al. 2011, Kerr et al. 2012, Zhang et al. 2012, Lei and Spradling 2013, Yuan et al. 2013) and required further evidence before believing the existence of OSCs and the idea that this work could be extended to other mammals, including humans (Telfer 2004). However, it is questionable whether studies using alternate species can be used to refute Tilly's claims in mice. Although the core question about the existence of these cells remains to many unanswered, even for those who believe in them, further questions must also be addressed, the most important of these being why do women undergo the menopause if these cells exist? Also, if not contributing to the number of primordial follicles when the population is running low, what is their function in ovarian biology? The use of OSCs, if functional, could not only revolutionise reproductive therapies (Dunlop et al. 2013), but also extend to prevention of post-menopausal health conditions, making the debate surrounding postnatal oogenesis even more significant.

\section{Do OSCs exist?}

The quest for these elusive OSCs in adult mammals was ignited by Tilly's work in 2004, and the subject has gained much momentum. The search has been fueled by the fact that invertebrates undergo postnatal oogenesis (Shim et al. 2014). The oogenesis cycle of Drosophila melanogaster, for example, is well understood, with postnatal oogenesis being well characterized (Lin \& Spradling 1997). Drosophila have a dynamic oogenesis process, with a new ovarian follicle being produced every $12 \mathrm{~h}$. Oocytes are produced continuously from germ cells throughout adulthood, as and when they are required, with homeostatic regulation based on nutritional and sperm availability. Oocytes do not stop being produced until the death of the animal, and thus Drosophila do not undergo menopause (Morris \& Spradling 2011).

Postnatal oogenesis occurs in Drosophila; however, they have little genetic similarity to humans. There is also evidence for postnatal oogenesis in prosimian primates, which are much more closely related to humans, raising the question of why humans and other mammals would evolve such a comparatively retrogressive reproductive mechanism. The strategy of producing all oocytes in fetal life, which requires preserving them potentially for decades (leaving them vulnerable to an accumulation of genetic damage) is risky, whereas, oocytes generated from stem cells would be a more resilient strategy. However, this evolutionary argument that postnatal oogenesis reduces the risk of cumulative genetic damage to oocytes is not evidence that there is a need for such cells in human fertility, let alone the existence of OSCs or any functionality they would provide.

\section{Does normal reproductive activity require OSCs?}

Scientists have attempted to demonstrate a physiological requirement for OSCs with mathematical models of the mammalian ovary. In 1987, the rate of atresia in the ovaries of adult mice of the CBA/Ca strain was studied, finding a significant rate of atresia despite constant follicle numbers. However, the peak of follicle atresia, studied using differential follicle counts in histologically sectioned ovaries, was detected at 20 days of age, with a decline in atresia after this period (Faddy et al. 1987). This peak of atresia at 20 days could indicate an instability in oocyte numbers in the early postnatal period that may later plateau and stabilise, and thus may not indicate a continued postnatal oogenesis (Faddy et al. 1987). This paper was not given the attention it deserved, and mathematical models of postnatal oogenesis were largely ignored until 2004. Tilly's group, who in 2004 controversially described the existence of OSCs in mammals, used mathematical modeling of immature follicle numbers as an initial argument for oogenesis in adult mice (Johnson et al. 2004). They found significant atresia of immature follicles in the adult mouse ovary, reaching a peak of 1200 dying follicles on day 42 of life. At such a high rate of atresia, the follicle reserve 
would not be expected to last through reproductive lifespan. Furthermore, when immature follicle numbers were assessed in mature mice, they were found to be less depleted than expected, with a rise in nonatretic follicles of $20 \%$ between day 4 and day 42 in one specific mouse strain, namely AKR/J. However, to try to minimize strain-specific results, changes in follicle numbers from birth to adulthood were studied in CD1 and C57BL/6 strains of mice, with comparable follicular dynamics. The difference in expected versus actual depletion was used to calculate the rate of neooogenesis required to maintain folliculogenesis: 77 oocytes per day (Johnson et al. 2004).

Following these unexpected results from Tilly's group, numerous other studies confirmed this constancy of follicular numbers in the prime reproductive period in mice (Kerr et al. 2006, Kerr et al. 2012) and humans (Bukovsky et al. 2004), but their explanations for it vary based on the level of atresia observed. The primordial follicle reserve pool in mouse ovaries from birth to 200 days was quantified (Kerr et al. 2006). The number of primordial follicles and oocytes did not significantly decrease between day 7 and day 100, despite migration and expulsion of hundreds of oocytes being observed, implying that the lack of decline must involve neooogenesis. Despite this conclusion, Kerr and coworkers and other groups subsequently produced data that imply that the lack of a decline in follicle numbers within the prime reproductive period simply represents a low rate of recruitment and atresia that makes losses statistically insignificant (Kerr et al. 2012, Lei \& Spradling 2013). These groups have proposed that the high rate of atresia reported in previous studies is an artifact of harsh fixation procedures and therefore the numbers of follicles undergoing atresia are overestimated (Byskov et al. 2005, Kerr et al. 2012). In addition, counting approaches vary between laboratories, which may cause technical differences in follicle numbers without any biological differences.

An additional complication in data comparison exists between studies because age and strain of mouse affect follicle dynamics (Byskov et al. 2005). For example, the CBA/Ca strain reported a significant decline in primordial follicle numbers between day 7 and day 100 (Faddy et al. 1987), which differs to the C57BL/6 strain as used by Kerr and coworkers (Kerr et al. 2006). However, as mentioned above, Tilly's group demonstrated that certain strains of mice have comparable follicle dynamics.

Interestingly, a recent study has demonstrated that there are two distinct waves of follicle development in mice, each with a different speed of follicle development (Zheng et al. 2014). Tamoxifen-inducible fluorescent proteins were used to differentially label the first, prepubertal wave of primordial follicles (induced at embryonic day (ED) 16.5) and adult primordial follicles (induced at postnatal day 5). Tracking of the labelled follicles as the mice developed indicated that follicles within the ovarian medulla were active at ED16.5, revealing a 'first wave', whereas cortical follicles were active and labelled when tamoxifen was injected at postnatal day (PD) 5, demonstrating an adult pool. Tracing the activity of each follicle pool through puberty into sexual maturity, the prepubertal follicle pool was initially dominant within the ovary for the first 6-7 weeks of life, facilitating the onset of puberty and sexual maturity. However, the 'first wave' follicles had dropped in proportion to $\sim 20 \%$ by PD60, revealing their rapid decline. In contrast, the adult follicle pool was developing slower than the prepubertal follicle pool and made up the majority of follicles from 6-7 weeks of age. The adult follicle pool also exhibited different growth dynamics, taking 37 days to develop from primordial to antral follicle, compared with 13 days for the 'first wave' (Zheng et al. 2014), however, it is yet to be determined whether this is due to intrinsic differences or external factors such as the endocrine environment. Nevertheless, when attempting to track the developmental dynamics of primordial follicles in adult mice, two distinct follicle pools with different growth rates would be a confounding variable for any mathematical calculation of oogenesis.

Although models of follicular dynamics based on mouse ovaries provide evidence supporting the presence of OSCs, studies of human ovarian reserves do not reach the same conclusions. Wallace and Kelsey generated a model of human ovarian reserve from conception to menopause based on the histological evidence available (Wallace \& Kelsey 2010). Any mathematical model that permitted an increase in numbers of non-growing follicles after 18-22 weeks, which would support the active functionality of OSCs, displayed a markedly inferior fit with the histological data than models that excluded this possibility. Therefore, this study, based on data from human ovaries, provides no evidence to support postnatal oogenesis in normal human physiology; however, it does not rule out a quiescent pool (Wallace \& Kelsey 2010). Difficulties in identifying and thus studying OSCs may account in part for the different conclusions drawn from different mathematical models of follicular dynamics.

\section{Are data describing OSCs comparable between species?}

As the breadth of OSC research has increased, many different species have been studied, but can the results of research from one species be applied to the oogenesis pattern of another? In addition to interspecies variation, large individual differences in follicle number creates variations in follicular quantification data in mice, which presents a confounding variable even for intraspecies research (Kerr et al. 2006). 
Animal research, with mice being the most widely studied animal in this field, allows in vivo investigation that can be more invasive enabling more manipulations than that is possible with humans. With the advancement of gene technology, for example, it has been possible to produce transgenic mice in which specific cell types are fluorescently labelled at specific time points (Zheng et al. 2014); this has revolutionised the specificity with which oogenesis can be studied and OSCs identified. However, follicular dynamics vary widely between mice and humans (White et al. 2012), which means that data based on mice should be applied to humans with caution.

Although mouse studies currently comprise the majority of the research, other animal species have also been used in the study of oogenesis, but not without limitations. Experiments on Drosophila and other invertebrates (Shim et al. 2014), demonstrate postnatal oogenesis, but these species lack the genetic similarity to allow direct comparison with mammals. Adult pigs have also been studied in relation to neo-oogenesis. In one such study, ovarian biopsies were taken and cultured, with cells within the cultured tissue expressing both stem and germ cell markers, a common method of OSC identification. These cells were therefore presumed to be OSCs (Bui et al. 2014). However, the fact remains that 'proof of neo-oogenesis in other mammals, such as prosimian primates, mice or pigs does not demonstrate the presence of neo-oogenesis in adult human ovaries' (Telfer 2004).

Human research has its own limitations. Due to ethical considerations, analysis of human ovaries is often limited to biopsies taken alongside a medical complaint, substantially limiting the amount of tissue that is available to be studied. The use of ovarian biopsies may not lead to the detection of OSCs even if they are present within the ovary, as the distribution of putative OSCs may not be uniform. Furthermore, most conditions that permit an ovarian biopsy may alter reproductive health from its normal physiological state; many of the samples are from anovulatory (VirantKlun et al. 2008) or dysfunctional ovaries that limits the understanding of postnatal oogenesis in normal human ovaries. However, whole ovaries from human participants of reproductive age undergoing gender reassignment have been studied (White et al. 2012), from which mitotically active germ cells were reported to be isolated. The use of whole ovaries as opposed to a small biopsy clearly has advantages, but only a small sample size was used in this study. Both biopsy and whole ovary sampling show a snapshot of the process of follicle development and potential oogenesis, which are highly dynamic and likely cyclical; effects of important factors such as the menstrual cycle cannot be measured, which limits understanding of the normal function any such cells may play within the complex environment of the human body. Importantly, the rate of any human neo-oogenesis is not known, and a small increase in the follicle reserve could be hard to detect with the sample sizes used. Such an increase could easily be hidden within statistical variance and assumed not to exist.

\section{Can we be sure we are identifying OSCs?}

As scientific research has progressed, the protocols with which researchers are able to identify and study OSCs have been revolutionised. Advances in genetics have been particularly invaluable in the identification of OSCs, as manipulations of the genome allow labelling of selected cells, providing the ability to follow specific cell lineages. Despite these advances in methodology, criticism remains surrounding the accuracy of these methods in specifically identifying OSCs.

Early studies investigating ovarian physiology used basic protocols such as haematoxylin and eosin staining (Arai 1920, Allen 1923). These studies made no attempt to individually or specifically identify OSCs, rendering their subsequent conclusions open to question. Markers of mitotic activity provide a mechanism of specifically identifying dividing cells (loannou 1967, Crone 1968, David 1974). For example, colchicine was injected (which arrests dividing cells in metaphase) into mice $9.5 \mathrm{~h}$ before tissue collection, allowing for the identification of mitotic cells (Bullough 1942). Although this method of identifying mitotic cells allowed specific detection of proliferating cells, the stem cells that produce somatic cells, such as granulosa and theca cells (Lavranos et al. 1999), act as a major confounding factor in any conclusions drawn from such data.

Proliferating somatic stem cells within the ovary are problematic if using mitotic markers alone to identify potential OSCs. Thus, a double labelling method using both germ cell markers and proliferating cell markers (usually BrdU or PCNA) can be used to avoid this issue and specifically label proliferating germ cells, i.e. OSCs. DEAD box polypeptide 4 (Ddx4), also referred to as Mouse Vasa homolog (MVH) is reported to be a specific marker for germ cells (Castrillon et al. 2000). The proteins encoded by $\mathrm{Ddx} 4$ play an important role in the germ cell development, although their exact role is unclear. Tilly's landmark 2004 study combined both $\mathrm{MVH}$ and BrdU labelling to identify proliferating germ cells within the ovaries of postnatal mice (Johnson et al. 2004). A different group used the same method in 2011 to identify OSCs collected from transgenic mice. The isolated cells were subsequently cultured and used to produce offsprings that displayed the same transgenic mutation as the mice they were collected from (Zhang et al. 2011). Ddx4 expression has subsequently been combined with fluorescent reporter activation in mice, stimulating fluorescence only in cells in which the Ddx4 gene promoter has been activated. These transgenic mice were used to identify cells in mouse ovaries that expressed $\mathrm{Ddx} 4$ and markers of 
primitive germ cells but not of mature oocytes; these actively proliferated in culture, thus demonstrating three characteristic features of OSCs; germ cell markers, lack of mature oocyte markers and proliferative potential (Park \& Tilly 2015).

The conclusion that OSCs have been identified depends on the 'belief' that Ddx4 proteins are only expressed within germ cells, allowing them to be differentiated from the somatic cells within the ovary. However, selective expression of $\mathrm{MVH} / \mathrm{Ddx} 4$ only in germ-line cells has been disputed; low levels of $\mathrm{MVH}$ expression have been found in the brain and kidney tissues of mice, which may demonstrate a more widespread expression than expected (Liu et al. 2007), potentially extending to one or more of the somatic cell lineages within the ovary. Further study of the specificity of $\mathrm{Ddx} 4$ expression is therefore required if we are to fully understand the expression profile of this supposed germ cell marker within the ovary, and thus its use in OSC identification. In addition, technical questions have been raised regarding immunohistochemical labelling of $\mathrm{MVH} ; \mathrm{MVH}$ is believed to be cytoplasmic in germ cells (Toyooka et al. 2000) and therefore it 'should not' be recognised using a surface label, which is precisely what is widely used. However, bioinformatics has predicted 2 potential transmembrane regions of the MVH protein (Zou et al. 2009), which would therefore be identified using immunohistochemistry, but this has yet to be confirmed. In fact, a 2016 study refuted the use of Ddx4 as an identifier of ovarian germ cells. Online transmembrane domain predictors were used on both the human and mouse Ddx4 protein, finding no evidence of a transmembrane region of human Ddx4 that could be used to isolate cells. One model predicted an extracellular domain in mice, but the overall evidence of a transmembrane $\mathrm{Ddx} 4$ region that could be isolated using antibodies was weak (Zarate-Garcia et al. 2016). Further investigation found that Ddx4 detection may be falsely identifying cells that do not express Ddx4 (ZarateGarcia et al. 2016). Interestingly, the same study also generated a total mouse ovarian cell suspension, from which cell surface Ddx4-positive cells were isolated and seeded for further analysis. Immunofluorescence showed some development of germ cell markers in the culture, but freshly isolated cells did not demonstrate these markers. Importantly, after 2-3 months of isolation the growth rate of cultures decreased and the cultures died, indicating a lack of immortality that is at odds with the definition of stem cells of any kind.

The identification of early meiosis markers within the ovary is provided as evidence of postnatal oogenesis, as initiation of meiosis would be required for oocyte production and follicle formation in adult life, and the process of meiosis only occurs within stem cells. These markers can be combined with BrdU/MVH double detection to provide further specificity to OSC identification (Johnson et al. 2004, Liu et al. 2007,
Bukovsky et al. 2008, Yuan et al. 2013). STRA8 is one such marker that indicates meiotic commitment. It was studied using gene expression profiling in both freshly isolated and cultured postnatal mouse OSCs (Imudia et al. 2013). Its absence in freshly isolated OSCs, but presence in cultured OSCs indicates a change in cell identity and potentially reflects the commitment to oocyte formation. However, the fact that this change in cell identity only occurs following culture implies that OSCs may be an anomaly arising from the culture itself. The absence of early meiotic markers in freshly isolated OSCs suggests that they may not undergo meiosis under native conditions in vivo, instead representing undifferentiated stem cells that are artificially stimulated to develop into germ cells following unphysiological culture. The 2004 Tilly paper used immunohistochemistry to identify postnatal cells expressing synaptonemal complex protein 3 (SCP3), Spo11 and Dmc1 in mice (Johnson et al. 2004), which are all meiotic markers. Interestingly, work from 2007 studied the presence of SCP3 within human adult ovarian samples and could not locate any meiocytes (Liu et al. 2007). However, this study was limited to the ovarian cortex, which may not be the location of OSCs. A significant drawback in the use of meiotic markers is the lack of understanding of potential OSC function. For example, OSCs may normally lie quiescent, in which case none of the meiotic or mitotic markers would be expressed, and thus these cells would remain undetectable in situ.

Cytoplasmic localisation for c-Kit, a stem cell marker which is a key regulator in germ cell differentiation but also present in somatic stem cells, is commonly used to provide further identification of potential OSCs in studies of postnatal mouse ovaries (Wright et al. 1996, Liu et al. 2007, Virant-Klun et al. 2008, Pacchiarotti et al. 2010, Parte et al. 2011). In combination with inconsistent with previous in paragraph and below localisation, oocyte proteins such as those from the zona pellucida (ZP) family have been detected to specifically identify OSCs and differentiate them from the stem cell precursors of theca or granulosa cells, which would be positive for c-kit detection alone (Bukovsky et al. 2004, BristolGould et al. 2006, Bukovsky et al. 2008, VirantKlun et al. 2008, Zou et al. 2009, Parte et al. 2011, Zhou et al. 2014). However, the ZP protein expression has only been detected in growing oocytes or follicular cells (Philpott et al. 1987, Sinowatz et al. 2001, Lan et al. 2004), and therefore the use of this as a marker of OSCs is dubious. C-kit positive cells have been identified using fluorescence immunohistochemistry on human ovarian scrapings from anovulatory women (Virant-Klun et al. 2008). Reverse transcriptase PCR was then used to identify the ZP2 expression, which was present in the c-kit positive cells. However, these cells did not express the meiotic marker SCP3, and thus would not be classified as OSCs in other studies. Although initial benchmarks 
have been suggested to identify in vitro derived germ cells (Handel et al. 2014), a universally accepted set of protocols to identify OSCs is not available, and is required to prevent discrepancies between studies that use different identification criteria for their cells. Until a universally accepted set of protocols is developed, cells identified as OSCs will be heterogeneous in nature and all subsequent testing and conclusions will be confounded by this.

The ability to specifically express visual markers, such as green fluorescent protein (GFP), in mouse models has allowed in vivo studies of putative OSCs. Initially, ovarian fragments from adult mice expressing GFP were grafted into wild-type ovaries and follicles were identified with either GFP positive oocytes and wild-type granulosa cells or vice versa, and were hailed as a demonstration of adult neo-oogenesis (Johnson et al. 2004). However, the generation of follicles containing cells from different sources has been suggested to be caused by follicular plasticity rather than postnatal oogenesis (Eppig \& Wigglesworth 2000, Albertini 2004) with the trauma of ovarian grafting stimulating a breakdown of follicles that reform in a different way.

AnalternategermcellmarkerOCT4 hasacharacterised expression profile through the stages of development in male germ-line stem cells. GFP expression was linked to OCT4 expression with the aim of identifying germline stem cells in ovaries (Pacchiarotti et al. 2010). Tracking of OCT4-driven GFP expression within the postnatal mouse ovary identified germ cells which were successfully cultured in vitro and induced to form embyroid bodies, demonstrating potential OSCs (Pacchiarotti et al. 2010). Tracking the proliferation and differentiation of Ddx4 expressing ovarian cells was carried out in vivo and in vitro (Zhang et al. 2012). Transgenic mice contained a Ddx4-Cre transgene that drove Cre recombinase expression in Ddx4 expressing cells, inducing recombination at a rainbow cassette region and thus switching GFP expression to red, orange or cyan fluorescent protein expression. Any cell expressing Ddx4, a marker of germ cells, was labelled with these coloured fluorescent proteins, whereas cells not expressing Ddx4, somatic cells, remain labelled with GFP. Using live cell imaging, no germ-line cells (as labelled with red, orange or cyan fluorescence) were found to divide in $72 \mathrm{~h}$ (Zhang et al. 2012). Subsequent conclusions were drawn questioning the existence of OSCs, but this study does not rule out the possibility of quiescent OSCs. Furthermore, a 72-h study period is not a sufficient length to take cyclical factors such as the menstrual cycle into account in OSC production. As with much of the research produced which appears to counter OSC existence, an absence of evidence is taken as evidence of absence. The lack of dividing OSCs within a study, especially one performed over a short period, does not provide sufficient evidence to conclude that they do not exist.

\section{Where should we look for OSCs?}

Many locations within the ovary have been proposed as the ovarian source of OSCs, with the ovarian surface epithelium (OSE) most prevalent. Numerous studies have located potential OSCs using immunohistochemistry from scrapings of OSE of human ovaries (Bukovsky et al. 2005, Virant-Klun et al. 2008, Parte et al. 2011, White et al. 2012), whereas studies taking ovarian cortex samples from both human (Liu et al. 2007) and monkey (Yuan et al. 2013) have not always identified the presence of these cells. However, the OSE is a single layer of cuboidal and flattened cells surrounding the ovary (Tan \& Fleming 2004), and is difficult to selectively scrape; therefore OSE samples are likely to have contamination from lower ovarian layers. A theory has been presented relating to this potential confound; OSCs may develop from progenitors in the underlying tunica albuginea via asymmetric division (Bukovsky et al. 2004), which is likely to be accidentally included in the majority of OSE samples. Work from this research group also suggests that epithelial crypts within ovaries may provide an additional source of OSCs (Bukovsky et al. 2004). However, further research into these areas of the ovary to specifically identify OSCs is required.

Another theory surrounding the origin of OSCs is that extraovarian cells might have a role in oogenesis, with evidence supporting this hypothesis first coming from Johnson and coworkers in 2005 (Johnson et al. 2005a). They demonstrated that the number of nonfollicle enclosed germ cells within the ovary (their classification of OSCs) was found to drop markedly in mice as they transitioned through puberty (Johnson et al. 2005a). The authors therefore looked for an alternative source of OSCs for postnatal oogenesis, and studied the potential role of bone marrow stem cells in the generation of oocytes. Immunofluorescent detection of $\mathrm{MVH}$ demonstrated the presence of germ-line markers in bone marrow cells, and further support came from the indication that oogenesis with GFP-positive oocytes could be stimulated in sterilised or genetically infertile wild-type mice following GFP-labelled bone marrow transplantation. However, these results were rapidly disputed, with suggestions that migrating cells or GFP leakage could be responsible for the results presented (Telfer et al. 2005) and no further work has been presented from the Tilly group relating to this hypothesis.

In addition, a study was published in 2006 exploring and refuting the proposal of extraovarian OSCs. Parabiotic mice were created to study any contribution of haematopoietic stem cells to the oocyte pool. A GFP transgenic mouse was surgically joined to a nontransgenic mouse, producing a common, anastamosed vasculature. After 6 months of parabiosis, GFP-labelled circulatory cells were detected within the ovary of the non-transgenic mouse and had associated with ovulating oocytes, but they did not develop any germ 
cell characteristics, instead demonstrating committed leukocyte characteristics. This led the authors to conclude that there is no evidence that circulating cells contribute to ovulated oocytes (Eggan et al. 2006). These criticisms do not, however, rule out the possibility that bone marrow cells indirectly support oocyte production, for example via more differentiated stromal cells (Johnson et al. 2005b). A monthly infusion of EGFP-labelled bone marrow cells from a young adult female mouse prevented normal reproductive failure of the ovaries of ageing female mice, without any offspring being EGFP positive (Selesniemi et al. 2009). This suggests a supportive role for bone marrow stem cells in the ovarian environment, without their direct development into oocytes. More research into this area, however, is needed before any concrete conclusions can be drawn. The question of OSC location may remain elusive until more is understood about the role that OSCs play under physiological conditions.

There is also the question of what role, if any, 'normal' initial gametogenesis and follicle nest formation plays in OSC development. The process of germ-cell nest formation and eventual breakdown to produce primordial follicles is highly conserved amongst different species (Pepling 2012), but in the context of OSC, this aspect appears to be completely surplus to their development.

\section{Do quiescent OSCs exist?}

While the physiological function of OSCs remains poorly understood, the study of these cells will remain limited and questions surrounding identification methods will remain unanswered. Studies have used mathematical, histological and ovary transplant methods to conclude that OSCs are actively dividing and undergoing meiosis in normal ovulating ovaries to maintain follicle numbers (Johnson et al. 2004, Bukovsky et al. 2005, Niikura et al. 2009, Zou et al. 2009, Parte et al. 2011, Zhang et al. 2011, White et al. 2012, Zhou et al. 2014). However, other studies, that have not found actively dividing germ cells, suggest a quiescent population of cells (Kerr et al. 2006, Liu et al. 2007, Kerr et al. 2012, Zhang et al. 2012, Lei \& Spradling 2013, Yuan et al. 2013). These quiescent cells may react only in response to specific alterations in signalling, for example in response to massive follicular depletion or when isolated in culture; alternatively, they may provide 'helper follicles' that are never ovulated but support the surviving follicles.

To test for a quiescent OSC population, 2 different in vivo methods have been applied; ovarian sterilisation with subsequent transplantation of labelled OSCs, or selective destruction of primordial follicles or oocytes. The latter method involves chemical destruction using doxorubicin (Johnson et al. 2004, Kerr et al. 2012) or trichostatinA (Kerretal.2012), or wholebody $\gamma$-irradiation
(Kerr et al. 2012). The theory behind follicle destruction was that it would stimulate any normally quiescent OSCs into activity after depletion of the follicle pool. However, the results from this experimental technique have come under severe scrutiny; results vary wildly in terms of primordial follicle numbers post treatment, and there is no evidence that these treatments do not damage OSCs (Johnson et al. 2004, Kerr et al. 2012). In 2014 , selective ablation of oocytes was used to provide evidence that no postnatal oogenesis occurs after oocyte destruction in transgenic mice (Zhang et al. 2014). The expression of growth differentiation factor 9 (GDF9) is specific to growing oocytes and not putative OSCs, and thus the generation of a model using the GDF9 promoter to stimulate expression of the diphtheria toxin receptor (DTR) (GDF9-Cre;iDTR mice) enables complete ablation of growing follicles without damage to potential OSCs. No oocytes were detected in the ovaries of these mice at 2,6 or 12 months post ablation, which does not support the hypothesis of a quiescent OSC population that is activated upon oocyte depletion (Zhang et al. 2014). However, there is no evidence that oocyte depletion would be the trigger to activate quiescent OSC cells; many other signals including hormonal changes could induce activation of this cell population.

The transplantation of isolated, cultured and GFPlabelled OSCs into sterilised mice has provided some very convincing results for the existence of adult OSCs and their function in restoring fertility. Histological ovary analyses and mating studies were performed to determine the outcome of the transplanted OSCs and their ability to restore fertility. Transplanted GFP-OSCs have not only gone on to develop into oocytes and form follicles, but have also been successfully fertilised to form transgenic offsprings, as assessed by expression of GFP (Zhang et al. 2011, Zhou et al. 2014). This method would provide the most convincing evidence yet for a population of OSCs that can develop into oocytes and produce offspring. However, the effects that culture and genetic manipulation can have on differentiation and cellular dynamics (Imudia et al. 2013) may reduce the validity of these results.

Also, there are questions surrounding the application of these cells to native animals and humans due to these extensive genetic changes and use of in vitro culture media. In vivo study of OSCs within normally functioning ovaries is necessary to understand their presence and function, quiescent or otherwise.

\section{What are the effects of external factors on OSCs?}

Many OSC studies require the cells to be separated from the animal and extensively manipulated. Despite the many advantages that these methods can offer, they overlook the importance of the ovarian and systemic environment on OSC development and differentiation. 
The menstrual cycle is a potential confounding variable that is often overlooked when OSC function is investigated, but analysis of both human and monkey ovarian samples at each stage of the menstrual cycle demonstrated that SCP3 was only readily detectable in the early luteal phase of the menstrual cycle, indicating that expression of meiotic markers may be dependent on the menstrual cycle (Bukovsky et al. 2008). Numerous samples from the tunica albuginea, ovarian surface epithelium and oocytes within primordial follicles from both human and monkey ovaries were assessed. Although the majority of samples were taken in the early luteal phase, with very few samples from other phases of the menstrual cycle, it still represents an interesting finding. OSC function may be influenced by the menstrual cycle, and this should be considered both in future studies and when interpreting past results. To control for this, the menstrual cycle phase should be noted for samples collected, and any manipulation compared with controls from the same phase of the cycle. The importance of the phase of the menstrual cycle on stem cells was indicated in a 2012 study into the influence of follicle stimulating hormone $(\mathrm{FSH})$ on mouse oogenesis. The levels of FSH vary in a cyclical manner according to the oestrous cycle, and the research demonstrated that gonadotropin treatment, mimicking the action of FSH, increased pluripotent stem cell activity in isolated ovaries, leading to increased meiosis and primordial follicle numbers (Bhartiya et al. 2012). However, the young age of the mice (1 month old, prepubertal) limits generalisation of these results. Following on from this initial research, the same group identified the presence of FSH receptor 3 (FSHR3) on sheep very small embryonic-like stem cells (VSELs) and revealed the receptor's function in stimulating FSHdriven development and differentiation of these cells into OSCs (Patel et al. 2013). However, extrapolating data to humans in relation to the complex action of FSH is confounded by differences in interspecies reproductive physiology.

In vitro studies have carried out extensive manipulation of OSCs, but the interpretation of these results must take into account the influence of any culture media. OSCs are assumed to be stem cells, and thus any alteration in signalling is bound to affect their subsequent development and differentiation. Although much research has gone into making culture media as physiologically similar to the normal ovarian milieu as possible, there remain divergences which could confound any results following OSC culture (Telfer \& Zelinski 2013). Media can contain unphysiological compounds such as phenol red and antibiotics. The effects of a combination of streptomycin and penicillin on culture and differentiation of murine embryonic stem cells into type II pneumocytes resulted in a $40 \%$ reduction in the growth rate of differentiating embryoid bodies (Cohen et al. 2006). This clear effect of antibiotics on cell culture needs to be considered when culturing putative OSCs. Culture conditions for oocytes and OSCs can also fail to replicate the cyclical nature of systemic factors such as FSH and the other hormones that exist in situ; for example, estradiol, anti-mullerian hormone and inhibins. These factors have a huge influence on the functioning of ovarian cells, and although their changing levels should ideally be accurately mimicked to follow physiological conditions, it is not possible to accurately replicate the in vivo environment in its entirety.

The dramatic effect of signals within culture media has been demonstrated with oestrogenic stimuli. Potential OSCs from adult human ovaries were cultured in the presence or absence of phenol red (a pro-oestrogenic stimulus). The presence of phenol red led to the generation of oocytes, as determined by immunohistochemical detection of the ZP protein and phase contrast microscopy, and the absence of it resulted in the generation of granulosa, epithelial and mesenchymal cells, identified morphologically (Bukovsky et al. 2005). The label of 'oocyte-like stem cells' may therefore be a misnomer; they may be bipotent progenitors for both granulosa cells and oocytes that develop differentially according to the signals received.

The majority of studies use feeder cells, which are typically inactivated fibroblasts, to initially culture OSCs (Virant-Klun etal.2008, Zou etal. 2009, Zhangetal.2011, Zhang et al. 2012, Zhou et al. 2014). No information is provided about potential interactions between the cultured cells and the feeder cells, or factors released by the feeder cells. For example, normal fibroblasts are known to produce aromatase (Berkovitz et al. 1984), which, if produced by the feeder cells, could interact with OSCs or oocytes within the culture medium and create a confounding variable.

The importance of the cellular environment within the ovary is often overlooked in relation to OSC culture. Granulosa and theca cells interact with oocytes, and would be likely to also interact with OSCs, to induce follicle formation, and also play key roles within the follicle itself. Metabolic cooperation has been discovered between granulosa cells and oocytes via gap junctions. This cooperation is crucial to normal oocyte development. Immunofluorescent labelling with phallodin identified transzonal projections (TZP) that project from granulosa cells through the ZP to form adherens junctions and gap junctions with the oocyte surface in cow oocytes (Li \& Albertini 2013). Further study of these junctions has indicated their importance in oocyte development; selective deletion of a gene that encodes an oocyte gap junction protein in mice, the Gja4 gene, arrests follicular development (Simon et al. 1997). Any similar interaction between granulosa or thecal cells and potential OSCs could be integral in differentiation into oocytes and initial follicle formation. 
Although the importance of hormonal signalling and cellular interaction with potential OSCs and follicular development has been demonstrated, the exact role of these external factors is unclear and is often not accounted for in OSC research. Studies to increase this understanding are likely to be invaluable in future OSC research and may even hold the key to definitive identification of these cells. OSCs may be present in ovaries as undifferentiated cells and many lines of research present data in favour of the presence of a stem cell capable of developing into a functioning oocyte. However, the unphysiological media used in vitro could direct the development of cultured cells artificially, whereas in vivo, the environment may play a different role altogether, or no role at all. Tracking of OSCs in vivo would not only demonstrate their existence, but any function that they play within the adult mammal.

\section{Why do women go through menopause?}

Irrespective of the research previously discussed, declining ovarian function remains a life-changing factor for every woman. Irrespective of OSC presence or function, women still go through the menopause and are affected in many ways; a complete loss of fertility and health problems associated with the menopause are common. This begs the question: if it is assumed that OSCs exist, why do women go through menopause? Many theories have been put forward to explain menopause assuming the presence of OSCs.

One theory is that OSCs are quiescent and never become activated in normal life; in which case, why are they there? Another is that the OSCs gradually lose their function, follicles can no longer be replenished and thus menopause occurs. Although stem cells are usually known for continuous replication, a study on the male equivalent of OSCs (type A spermatogonia) has questioned this for germ-line stem cells. The testes of aged mice were studied, and by 2 years of age, $75 \%$ of testes were atrophied, with a dramatic decline in the number, activity and quality of germ-line stem cells (Zhang et al. 2006). This process of declining activity may occur in potential OSCs, which are likened morphologically to type A spermatogonia (Zou et al. 2009); this would explain cessation of ovulation. Stem cell function has been shown to decline with age, associated closely with changes in cellular physiology, gene expression and environment (Signer 2013). Decline in stem cell function could impact not only OSCs, but also granulosa/theca stem cells, which play a crucial role in follicle development.

A third theory is that the OSCs are still able to differentiate into functioning oocytes, but granulosa or theca cell numbers are limited, and this is what stops follicular development and initiates menopause by causing a deterioration of the somatic microenvironment
(Tilly \& Telfer 2009). Metabolic cooperation between granulosa cells and oocytes plays a crucial role in the follicle formation, and disruption of this process could provide an explanation for menopause. It has been observed that the numbers of oocyte-like cells are present in excess of the supporting granulosa cells in sections of adult human ovaries (Bukovsky et al. 2004). This imbalance may prevent an adequate number of TZPs forming, thus arresting the follicular development and preventing oocyte development, but further study is crucial to indicate whether this mismatch is actually a limiting factor in follicular development. Both granulosa and theca cell dynamics and development are relatively understudied, and future experiments on these cells could shed important light on menopause.

A fourth theory suggests that an age-related change in local and systemic signals could explain declining ovarian function without ruling out the presence of OSCs. A change in growth factor or hormonal signals could stop OSC development and cause depletion of the follicle pool. The addition of GFP-expressing OSCs from oocyte deplete aged mice ovaries into the ovarian bursal sacs of young recipient mice caused their differentiation into oocytes (Niikura et al. 2009). This indicates an age-related change in the environment that could negatively affect OSC development and differentiation. If hormonal signals play a role, comparison of normal post-menopausal ovaries and those subjected to hormone replacement therapy would be interesting, though availability of tissue is very limited. Follicular dynamics have been linked to the immune system and bone marrow, with immunohistochemical studies linking differentiation of the OSE with macrophage and Thy- 1 glycoprotein levels, an immune system morphoregulatory molecule (Bukovský et al. 1995). The immune system is known to decline in an age-related manner, and further research into the connection between the immune system and ovarian dynamics could indicate a reason for ovarian decline, potentially by reduced development of the OSE.

\section{How could we use OSCs therapeutically?}

If these cells are definitively identified and their functions confirmed, their manipulation could present a major breakthrough in fertility treatments and post-menopausal health, for example, for women suffering from primary ovarian insufficiency (POI) or, as previously known, premature ovarian failure (POF) (Dunlop et al. 2013). As data is accumulating to support the presence of a cell capable of developing functional oocytes in vitro, defining the function of such cells in vivo is crucial. Introducing or activating OSCs within the ovary could restore the function of the ovary, reducing the number of women suffering from post-menopausal health conditions. From a fertility perspective, OSCs removed 
from women about to undergo ovotoxic procedures could be isolated and cryopreserved and then be injected back into the ovary post-procedure. Cryopreservation of presumed OSCs has been demonstrated not to affect their proliferative or differentiation capacity (Zou et al. 2009), and thus could provide many women with the ability to produce their own egg, with a view to carrying their own child after ovotoxic treatments. Further details on the potential for fertility preservation is described in recent reviews (Dunlop et al. 2013, Bukovsky 2015). However, care must be taken if treatment for infertility involving OSC cryopreservation and re-implantation were to be extended to age-related ovarian decline, as the oocytes produced from OSCs from older women may be genetically damaged, increasing the risk of complications.

The American company OvaScience (http://www. ovascience.com/treatments) has translated the potential of postnatal oogenesis into preclinical trials, which claim to grow 'egg precursor' cells in vitro and replace them back into the same ovary. Although an exciting transition from theory into practical application, these treatments have remained preclinical for several years, indicating that much more needs to be understood before fertility treatments employing OSCs can be fruitful, if ever.

The ovarian environment must also be considered in relation to fertility restoration. A pilot study presented at the 2013 annual meeting of the European Society of Human Reproduction and Embryology (ESHRE) described potential OSCs isolated and propagated from normal women and women with Turner's syndrome (TS) ovaries (Anderson 2013). The propagated cells were GFP labelled and injected into both normal and TS human ovarian tissue in vitro. In the normal ovarian tissue, both the TS or normal OSCs generated follicles containing GFP positive 'oocytes', however, in the TS ovarian tissue, neither the normal or TS OSCs formed follicles indicating that viable OSCs require a viable environment to develop. Further study of the wider ovarian environment, including somatic cells as well as signalling, must be conducted to provide context for OSC development.

Most interestingly, a recent study by Telfer's group has revealed that a specific regime of chemotherapy actually seems to stimulate the numbers of primordial germ cells in female human ovaries (McLaughlin et al. 2017). They observed that a regime of ABVD (adriamycin, bleomycin, vinblastine and dacarbazine) administered to patients with Hodgkins lymphoma (HL) led to an increase in mean follicular density compared to samples from patients treated with an alternate regime for HL (OEPACOPDAC: combined vincristine, etoposide, prednisone, doxorubicin (OEPA) and cyclophosphamide, vincristine, prednisone, dacarbazine (COPDAC)) and untreated controls. However, despite the presence of more follicles in the ovaries treated with ABVD, the proportion of follicles that developed to the secondary stage was lower than the control samples (McLaughlin et al. 2017) indicating that there is still much to learn about these cells and their potential to become developmentally competent oocytes.

However, is the subject of OSCs already old news when we consider therapeutic treatments? A 2016 study used adult mouse fibroblasts to generate induced pluripotent stem cells (iPSCs) that were directed in vitro to reconstitute oogenesis (Hikabe et al. 2016). The oocytes were matured and fertilized, ultimately producing mouse pups without any apparent abnormalities. This technology bypasses the ovary and potential OSCs entirely, and raises the question of whether OSCs would be used (if isolated), or whether iPSCs could be manipulated for the same potential benefits.

\section{Conclusion}

The field of postnatal oogenesis is controversial and highly polarised, many scientists believe it to be the next big breakthrough in reproductive biology, others believe in the existence of OSCs but are sceptical about their role in functional oocyte development and others believe that OSCs do not exist and that 'belief' in their existence arises from misinterpretation of ambiguous results or culture. Compelling evidence for postnatal neo-oogenesis exists, however, as always, further research is needed to focus on the interactions between OSCs and the wider ovarian environment. This will provide the greater understanding and context needed to definitively prove the existence, function and potential usefulness of these elusive cells.

\section{Declaration of interest}

The authors declare that there is no conflict of interest that could be perceived as prejudicing the impartiality of this review.

\section{Funding}

This research did not receive any specific grant from any funding agency in the public, commercial or not-for-profit sector.

\section{References}

Albertini DF 2004 Micromanagement of the ovarian follicle reservedo stem cells play into the ledger? Reproduction 127 513-514. (doi:10.1530/rep.1.00247)

Allen E 1923 Ovogenesis during sexual maturity. American Journal of Anatomy 31 439-481. (doi:10.1002/aja.1000310502)

Anderson RA 2013 Evaluation of oogonial stem cells and neo-oogenesis in ovaries of girls and women with Turner syndrome: a pilot study. Human Reproduction 28 52-55. (doi:10.1093/humrep/det165)

Arai H 1920 On the postnatal development of the ovary (albino rat), with especial reference to the number of ova. American Journal of Anatomy 27 405-462. (doi:10.1002/aja.1000270403) 
Berkovitz GD, Fujimoto M, Brown TR, Brodie AM \& Migeon CJ 1984 Aromatase activity in cultured human genital skin fibroblasts. Journal of Clinical Endocrinology and Metabolism 59 665-671.

Bhartiya D, Sriraman K, Gunjal P \& Modak H 2012 Gonadotropin treatment augments postnatal oogenesis and primordial follicle assembly in adult mouse ovaries? Journal of Ovarian Research 5 32. (doi:10.1186/17572215-5-32)

Brinster RL 2007 Male germline stem cells: from mice to men. Science $\mathbf{3 1 6}$ 404-405. (doi:10.1126/science.1137741)

Bristol-Gould SK, Kreeger PK, Selkirk CG, Kilen SM, Mayo KE, Shea LD \& Woodruff TK 2006 Fate of the initial follicle pool: empirical and mathematical evidence supporting its sufficiency for adult fertility. Developmental Biology 298 149-154. (doi:10.1016/j. ydbio.2006.06.023)

Bui HT, Van Thuan N, Kwon DN, Choi YJ, Kang MH, Han JW, Kim T, \& Kim JH 2014 Identification and characterization of putative stem cells in the adult pig ovary. Development 141 2235-2244. (doi:10.1242/ dev.104554)

Bukovsky A 2011 How can female germline stem cells contribute to the physiological neo-oogenesis in mammals and why menopause occurs? Microscopy and Microanalysis 17 498-505. (doi:10.1017/ S143192761000036X)

Bukovsky A 2015 Novel methods of treating ovarian infertility in older and POF women, testicular infertility, and other human functional diseases. Reproductive Biology and Endocrinology 13 1. (doi:10.1186/14777827-13-1)

Bukovsky A, Caudle MR, Gupta SK, Svetlikova M, Selleck-White R, Ayala AM, \& Dominguez R 2008 Mammalian neo-oogenesis and expression of meiosis-specific protein SCP3 in adult human and monkey ovaries. Cell Cycle 7 683-686. (doi:10.4161/cc.7.5.5453)

Bukovsky A, Caudle MR, Svetlikova M, \& Upadhyaya NB 2004 Origin of germ cells and formation of new primary follicles in adult human ovaries. Reproductive Biology and Endocrinology 2 20. (doi:10.1186/14777827-2-20)

Bukovský A, Keenan JA, Caudle MR, Wimalasena J, Upadhyaya NB \& Van Meter SE 1995 Immunohistochemical studies of the adult human ovary: possible contribution of immune and epithelial factors to folliculogenesis. American Journal of Reproductive Immunology 33 323-340. (doi:10.1111/j.1600-0897.1995.tb00901.x)

Bukovsky A, Svetlikova M \& Caudle MR 2005 Oogenesis in cultures derived from adult human ovaries. Reproductive Biology and Endocrinology 3 17. (doi:10.1186/1477-7827-3-17)

Bullough W 1942 Oogenesis and its relation to the oestrous cycle in the adult mouse. Journal of Endocrinology 3 141-149. (doi:10.1677/ joe.0.0030141)

Byskov AG, Faddy MJ, Lemmen JG \& Andersen CY 2005 Eggs forever? Differentiation; Research in Biological Diversity 73 438-446. (doi:10.1111/j.1432-0436.2005.00045.x)

Byskov AG, Høyer PE, Yding Andersen C, Kristensen SG, Jespersen A \& Møllgård K 2011 No evidence for the presence of oogonia in the human ovary after their final clearance during the first two years of life. Human Reproduction 26 2129-2139. (doi:10.1093/humrep/der145)

Castrillon DH, Quade BJ, Wang TY, Quigley C \& Crum CP 2000 The human VASA gene is specifically expressed in the germ cell lineage. PNAS 97 9585-9590. (doi:10.1073/pnas.160274797)

Cohen S, Samadikuchaksaraei A, Polak JM \& Bishop AE 2006 Antibiotics reduce the growth rate and differentiation of embryonic stem cell cultures. Tissue Engineering 12 2025-2030. (doi:10.1089/ten.2006.12.2025)

Crone MP \& Peters H 1968 Unusual incorporation of tritiated thymidine into early diplotene oocytes of mice. Experimental Cell Research $\mathbf{5 0}$ 664-668. (doi:10.1016/0014-4827(68)90432-1)

David GF, Anand Kumar TC \& Baker TC. 1974 Uptake of tritiated thymidine by primordial germ cells in the ovaries of the adult slender loris. Journal of Reproduction and Fertility 41 447-451. (doi:10.1530/jrf.0.0410447)

Dunlop CE, Telfer EE \& Anderson RA 2013 Ovarian stem cells-potential roles in infertility treatment and fertility preservation. Maturitas $\mathbf{7 6}$ 279-283. (doi:10.1016/j.maturitas.2013.04.017)

Eggan K, Jurga S, Gosden R, Min IM \& Wagers AJ 2006 Ovulated oocytes in adult mice derive from non-circulating germ cells. Nature $\mathbf{4 4 1}$ 1109-1114. (doi:10.1038/nature04929)

Eppig JJ \& Wigglesworth K 2000 Development of mouse and rat oocytes in chimeric reaggregated ovaries after interspecific exchange of somatic and germ cell components. Biology of Reproduction 63 1014-1023. (doi:10.1095/biolreprod63.4.1014)

Esmaeilian Y, Atalay A \& Erdemli E 2015 Post-natal oogenesis: a concept for controversy that intensified during the last decade. Zygote 23 315-326. (doi: 10.1017/S0967199413000622).

Faddy MJ, Telfer E \& Gosden RG 1987 The kinetics of pre-antral follicle development in ovaries of CBA/Ca mice during the first 14 weeks of life. Cell and Tissue Kinetics 20 551-560. (doi:10.1111/j.1365-2184.1987. tb01364.x)

Gosden R 2004 Germline stem cells in the postnatal ovary: is the ovary more like a testis? Human Reproduction Update 10 193-195. (doi:10.1093/ humupd/dmh023)

Handel MA, Eppig J \& Schimenti JC 2014 Applying "gold standards" to in-vitro-derived germ cells. Cell 157 1257-1261. (doi:10.1016/j. cell.2014.05.019)

Hansen KR, Knowlton NS, Thyer AC, Charleston JS, Soules MR \& Klein NA 2008 A new model of reproductive aging: the decline in ovarian nongrowing follicle number from birth to menopause. Human Reproduction 23 699-708. (doi:10.1093/humrep/dem408)

Hikabe O, Hamazaki N, Nagamatsu G, Obata Y, Hirao Y, Hamada N, Shimamoto S, Imamura T, Nakashima K, Saitou M et al. 2016 Reconstitution in vitro of the entire cycle of the mouse female germ line. Nature 539 299-303. (doi:10.1038/nature20104)

Imudia AN, Wang N, Tanaka Y, White YAR, Woods DC \& Tilly JL 2013 Comparative gene expression profiling of adult mouse ovary-derived oogonial stem cells supports a distinct cellular identity. Fertility and Sterility 100 1451-1458.e2. (doi: 10.1016/j.fertnstert.2013.06.036).

Ioannou JM 1967 Oogenesis in adult prosimians. Journal of embryology and experimental morphology 17 139-145.

Johnson J, Bagley J, Skaznik-Wikiel M, Lee HJ, Adams GB, Niikura Y, Tschudy KS, Tilly JC, Cortes ML, Forkert R et al. 2005a Oocyte generation in adult mammalian ovaries by putative germ cells in bone marrow and peripheral blood. Cell 122 303-315. (doi:10.1016/j.cell.2005.06.031)

Johnson J, Canning J, Kaneko T, Pru JK \& Tilly JL 2004 Germline stem cells and follicular renewal in the postnatal mammalian ovary. Nature $\mathbf{4 2 8}$ 145-150. (doi:10.1038/nature02316)

Johnson J, Skaznik-Wikiel M, Lee H-J, Niikura Y, Tilly JC \& Tilly JL 2005b Setting the record straight on data supporting postnatal oogenesis in female mammals. Cell Cycle 4 1471-1477.

Kerr JB, Brogan L, Myers M, Hutt KJ, Mladenovska T, Ricardo S, Hamza K, Scott CL, Strasser A \& Findlay JK 2012 The primordial follicle reserve is not renewed after chemical or -irradiation mediated depletion. Reproduction 143 469-476. (doi:10.1530/REP-11-0430)

Kerr JB, Duckett R, Myers M, Britt KL, Mladenovska T \& Findlay JK 2006 Quantification of healthy follicles in the neonatal and adult mouse ovary: evidence for maintenance of primordial follicle supply. Reproduction 132 95-109. (doi:10.1530/rep.1.01128)

Lan ZJ, Xu X \& Cooney AJ 2004 Differential oocyte-specific expression of Cre recombinase activity in GDF-9-iCre, Zp3cre \& Msx2Cre transgenic mice. Biology of Reproduction 71 1469-1474. (doi:10.1095/ biolreprod.104.031757)

Lavranos TC, Mathis JM, Latham SE, Kalionis B, Shay JW \& Rodgers RJ 1999 Evidence for ovarian granulosa stem cells: telomerase activity and localization of the telomerase ribonucleic acid component in bovine ovarian follicles. Biology of Reproduction 61 358-366. (doi:10.1095/ biolreprod61.2.358)

Lei L \& Spradling AC 2013 Female mice lack adult germ-line stem cells but sustain oogenesis using stable primordial follicles. PNAS 110 8585-8590. (doi:10.1073/pnas.1306189110)

Li R \& Albertini DF 2013 The road to maturation: somatic cell interaction and self-organization of the mammalian oocyte. Nature Reviews Molecular Cell Biology 14 141-152. (doi:10.1038/nrm3531)

Lin H \& Spradling AC 1997 A novel group of pumilio mutations affects the asymmetric division of germline stem cells in the Drosophila ovary. Development 124 2463-2476.

Liu Y, Wu C, Lyu Q, Yang D, Albertini DF, Keefe DL \& Liu L 2007 Germline stem cells and neo-oogenesis in the adult human ovary. Developmental Biology 306 112-120. (doi:10.1016/j.ydbio.2007.03.006)

McLaughlin M, Kelsey TW, Wallace WH, Anderson RA \& Telfer EE 2017 Non-growing follicle density is increased following adriamycin, bleomycin, vinblastine and dacarbazine (ABVD) chemotherapy in the adult human ovary. Human Reproduction 32 165-174. 
Morris LX \& Spradling AC 2011 Long-term live imaging provides new insight into stem cell regulation and germline-soma coordination in the Drosophila ovary. Development 138 2207-2215. (doi:10.1242/ dev.065508)

Niikura Y, Niikura T \& Tilly JL 2009 Aged mouse ovaries possess rare premeiotic germ cells that can generate oocytes following transplantation into a young host environment. Aging 1 971-978. (doi:10.18632/ aging.100105)

Pacchiarotti J, Maki C, Ramos T, Marh J, Howerton K, Wong J, Pham J, Anorve S, Chow YC \& Izadyar F 2010 Differentiation potential of germ line stem cells derived from the postnatal mouse ovary. Differentiation 79 159-170. (doi:10.1016/j.diff.2010.01.001)

Park ES \& Tilly J 2015 Use of DEAD-box polypeptide-4 (Ddx4) gene promoter-driven fluorescent reporter mice to identify mitotically active germ cells in post-natal mouse ovaries. Molecular Human Reproduction 21 58-65. (doi:10.1093/molehr/gau071)

Parte S, Bhartiya D, Telang J, Daithankar V, Salvi V, Zaveri K \& Hinduja I 2011 Detection, characterization, and spontaneous differentiation in vitro of very small embryonic-like putative stem cells in adult Mammalian ovary. Stem Cells and Development 20 1451-1464. (doi:10.1089/ scd.2010.0461)

Patel H, Bhartiya D, Parte S, Gunjal P, Vedulkar S \& Bhatt M 2013 Follicle stimulating hormone modulates ovarian stem cells through alternately spliced receptor variant FSH-R3. Journal of Ovarian Research 652 (doi:10.1186/1757-2215-6-52)

Pearl R \& Schoppe WE 1921 Studies on the physiology of reproduction in the domestic fowl. Journal of Experimental Zoology 34 101-118.

Pepling ME 2012 Follicular assembly: mechanisms of action. Reproduction 143 139-149. (doi:10.1530/REP-11-0299)

Peters H, Levy E \& Crone M 1962 Deoxyribonucleic acid synthesis in oocytes of mouse embryos. Nature 195 915-916. (doi:10.1038/195915a0)

Philpott CC, Ringuette MJ \& Dean J 1987 Oocyte-specific expression and developmental regulation of ZP3, the sperm receptor of the mouse zona pellucida. Developmental Biology 121 568-575. (doi:10.1016/00121606(87)90192-8)

Selesniemi K, Lee H-J, Niikura T \& Tilly JL 2009 Young adult donor bone marrow infusions into female mice postpone age-related reproductive failure and improve offspring survival. Aging 1 49-57. (doi:10.18632/ aging.100002)

Shim HJ, Lee E-M, Nguyen LD, Shim J \& Song Y-H 2014 High-dose irradiation induces cell cycle arrest, apoptosis, and developmental defects during Drosophila oogenesis. PLOS ONE 9 e89009. (doi:10.1371/ journal.pone.0089009)

Signer RM \& Morrison SJ. 2013 Mechanisms that regulate stem cell aging and lifespan. Cell Stem Cell 12 152-165. (doi:10.1016/j. stem.2013.01.001)

Simon AM, Goodenough DA, Li E \& Paul DL 1997 Female infertility in mice lacking connexin 37. Nature 385 525-529. (doi:10.1038/385525a0)

Sinowatz F, Kolle S \& Topfer-Petersen E 2001 Biosynthesis and expression of zona pellucida glycoproteins in mammals. Cells Tissues Organs 168 24-35. (doi:10.1159/000016803)

Tan OL \& Fleming JS 2004 Proliferating cell nuclear antigen immunoreactivity in the ovarian surface epithelium of mice of varying ages and total lifetime ovulation number following ovulation. Biology of Reproduction 71 1501-1507. (doi:10.1095/ biolreprod.104.030460)

Telfer EE 2004 Germline stem cells in the postnatal mammalian ovary: A phenomenon of prosimian primates and mice? Reproductive Biology and Endocrinology 224

Telfer EE, Gosden RG, Byskov AG, Spears N, Albertini D, Andersen CY, Anderson R, Braw-Tal R, Clarke H, Gougeon A et al. 2005 On regenerating the ovary and generating controversy. Cell 122 821-822. (doi:10.1016/j.cell.2005.09.004)

Telfer EE \& Zelinski MB 2013 Ovarian follicle culture: advances and challenges for human and nonhuman primates. Fertility and Sterility $\mathbf{9 9}$ 1523-1533. (doi:10.1016/j.fertnstert.2013.03.043)

Tilly JL \& Telfer EE 2009 Purification of germline stem cells from adult mammalian ovaries: a step closer towards control of the female biological clock? Molecular Human Reproduction 15 393-398. (doi:10.1093/ molehr/gap036)

Toyooka Y, Tsunekawa N, Takahashi Y, Matsui Y, Satoh M \& Noce T 2000 Expression and intracellular localization of mouse Vasa-homologue protein during germ cell development. Mechanisms of Development 93 139-149. (doi:10.1016/S0925-4773(00)00283-5)

Virant-Klun I, Zech N, Rozman P, Vogler A, Cvjeticanin B, Klemenc P, Malicev E \& Meden-Vrtovec H 2008 Putative stem cells with an embryonic character isolated from the ovarian surface epithelium of women with no naturally present follicles and oocytes. Differentiation; Research in Biological Diversity 76 843-856. (doi:10.1111/j.14320436.2008.00268.x)

Waldeyer W 1870 Eirstock und Ei. Germany: Engelmann.

Wallace WH \& Kelsey TW 2010 Human ovarian reserve from conception to the menopause. PLOS ONE $\mathbf{5}$ e8772. (doi:10.1371/journal. pone.0008772)

White YAR, Woods DC, Takai Y, Ishihara O, Seki H \& Tilly JL 2012 Oocyte formation by mitotically active germ cells purified from ovaries of reproductive-age women. Nature Medicine 18 413-421. (doi:10.1038/ $\mathrm{nm}$.2669)

Wright WE, Piatyszek MA, Rainey WE, Byrd W \& Shay JW 1996 Telomerase activity in human germline and embryonic tissues and cells. Developmental Genetics 18 173-179. (doi:10.1002/(SICI)15206408(1996)18:2<173::AID-DVG10>3.0.CO;2-3)

Yuan J, Zhang D, Wang L, Liu M, Mao J, Yin Y, Ye X, Liu N, Han J, Gao $\mathbf{Y}$ et al. 2013 No evidence for neo-oogenesis may link to ovarian senescence in adult monkey. Stem Cells 31 2538-2550. (doi:10.1002/ stem.1480)

Zarate-Garcia L, Lane SIR, Merriman JA \& Jones KT 2016 FACS-sorted putative oogonial stem cells from the ovary are neither DDX4-positive nor germ cells. Scientific Reports 6 27991. (doi:10.1038/srep27991)

Zhang H, Liu L, Li X, Busayavalasa K, Shen Y, Hovatta O, Gustafsson J-Å \& Liu K 2014 Life-long in vivo cell-lineage tracing shows that no oogenesis originates from putative germline stem cells in adult mice. PNAS 111 17983-17988. (doi:10.1073/pnas.1421047111)

Zhang H, Zheng W, Shen Y, Adhikari D, Ueno H \& Liu K 2012 Experimental evidence showing that no mitotically active female germline progenitors exist in postnatal mouse ovaries. PNAS 109 12580-12585. (doi:10.1073/ pnas.1206600109)

Zhang, X, KT Ebata, B Robaire \& MC Nagano 2006 Aging of male germ line stem cells in mice. Biology of Reproduction 74 119-124. (doi:10.1095/ biolreprod.105.045591)

Zhang Y, Yang Z, Yang Y, Wang S, Shi L, Xie W, Sun K, Zou K, Wang L, Xiong J et al. 2011 Production of transgenic mice by random recombination of targeted genes in female germline stem cells. Journal of Molecular Cell Biology 3 132-141. (doi:10.1093/jmcb/mjq043)

Zheng W, Zhang H, Gorre N, Risal S, Shen Y \& Liu K 2014 Two classes of ovarian primordial follicles exhibit distinct developmental dynamics and physiological functions. Human Molecular Genetics 23 920-928. (doi: 10.1093/hmg/ddt486)

Zhou L, Wang L, Kang JX, Xie W, Li X, Wu C, Xu B \& Wu J 2014 Production of fat- 1 transgenic rats using a post-natal female germline stem cell line. Molecular Human Reproduction 20 271-281. (doi:10.1093/molehr/ gat081)

Zou K, Yuan Z, Yang Z, Luo H, Sun K, Zhou L, Xiang J, Shi L, Yu Q, Zhang Y et al. 2009 Production of offspring from a germline stem cell line derived from neonatal ovaries. Nature Cell Biology 11 631-636. (doi:10.1038/ncb1869)

Zuckerman S 1951 The number of oocytes in the mature ovary. Recent Progress in Hormone Research 6 63-108.

Received 5 January 2017

First decision 28 February 2017

Revised manuscript received 29 March 2017

Accepted 7 April 2017 